\title{
Models of fragment penetration and fireball evolution
}

\author{
By DAVID A. CRAWFORD \\ Experimental Impact Physics Department, Sandia National Laboratories, MS 0821, \\ Albuquerque, NM 87185, USA
}

\begin{abstract}
A new analytical model that is calibrated against numerical simulations performed with the CTH shock physics code provides a useful description of the entry of Periodic Comet ShoemakerLevy 9 into the Jovian atmosphere. Mass loss due to radiative heating of fragments larger than $100 \mathrm{~m}$ in diameter is insignificant because of energy conservation during the ablative process. Nevertheless, radiative ablation is a major contributor to atmospheric energy deposition at high altitude and plays an important role in early-time fireball evolution. The analytical model provides the initial conditions from which fireball and plume evolution can be calculated using CTH. The results from these simulations suggest that if the tops of the plumes originated from a specific level of the Jovian atmosphere then maximum plume heights are independent of fragment size provided the fragments penetrated at least $30 \mathrm{~km}$ below this level. If the tops of the plumes originated from the visible cloud tops, then fragment masses greater than $4 \times 10^{12} \mathrm{~g}$, corresponding to $200 \mathrm{~m}$ diameter fully dense water ice, are required to explain the observations. If the plumes originated from the $\mathrm{NH}_{4} \mathrm{SH}$ layer then masses greater than $3 \times 10^{13} \mathrm{~g} \mathrm{(400} \mathrm{m}$ water ice) are required. The lateral extent and mass of the observable plume are functions of fragment size and contribute to the lateral extent and albedo of the debris patterns after re-impact with the atmosphere. The apparent gap between the central disturbance of the impact site and the inner front of the crescent-shaped ejecta may reflect the fragment's depth of penetration below the source layer of the visible ejecta.
\end{abstract}

\section{Introduction}

Models of Comet Shoemaker-Levy 9 fragments entering the Jovian atmosphere and subsequent fireball and plume evolution are strongly constrained by the wealth of consequences observed during the impact week. Some consequences were predicted. For example, three-dimensional, bilaterally-symmetric computational simulations performed prior to the impacts demonstrated that each event would produce a debris-laden fireball/plume that would expand explosively up the entry channel produced by the impacting fragment (Boslough et al. 1994a,b; Crawford et al. 1994, 1995b; Takata et al. 1994; Shoemaker et al. 1995). The simulations were consistent with the bilateral symmetry of the observed impact sites, the concentration of dark ejecta materials in the direction from which the fragments came and the plumes themselves, which exhibited a lateral offset during their evolution (Figure 1). Other consequences, such as the strength of the 'main' infrared event that correlated with the splashback of plume materials onto the atmosphere, were unanticipated, but consistent with pre-impact theoretical models (Figure 2). Still other consequences were entirely unexpected. For example, plumes observed by the Hubble Space Telescope all had approximately the same maximum altitude yet the dark ejecta they left behind varied considerably in albedo and lateral extent (Hammel et al. 1995). Several proposals have been made to explain this phenomenon (see the chapter by Zahnle and later in this chapter) but the matter is not yet settled.

Pre-impact and post-impact theoretical modeling can help provide a framework for interpreting the observations. Figure 3 depicts an idealized representation of the sequence of events inferred from Earth-based photometry data and is based, in part, on computational simulations. The figure is not intended to show the exact geometry, but 


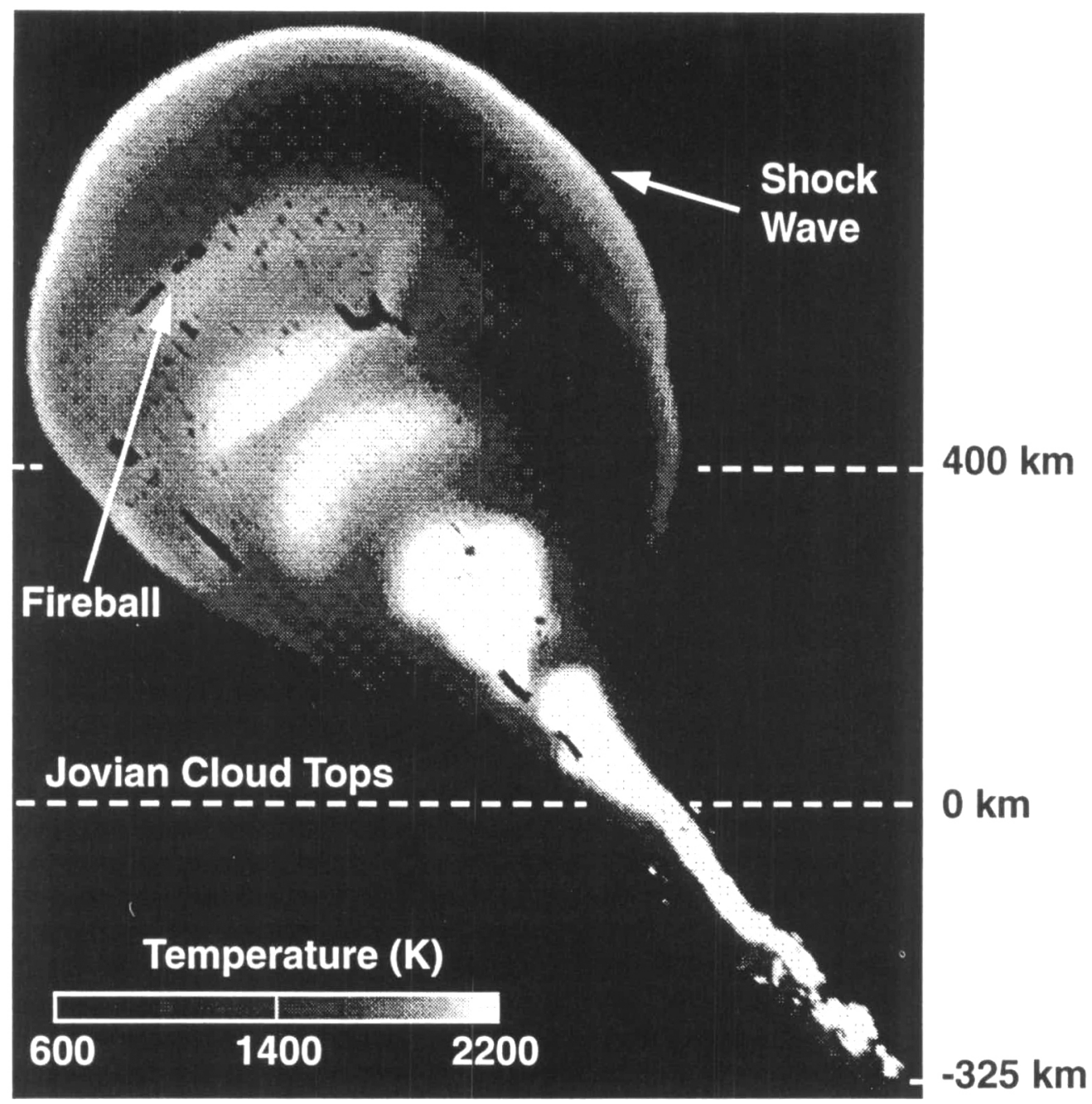

Figure 1. Pre-impact three-dimensional simulation of the fireball produced from a $3 \mathrm{~km}$ ice fragment entering the Jovian atmosphere at $60 \mathrm{~km} \mathrm{~s}^{-1}$. This is a two-dimensional slice along the bilateral symmetry plane made 69 seconds after the passage of the fragment through the $100 \mathrm{~km}$ altitude referenced to the 1-bar pressure level of the atmosphere. Gray scale represents temperature. The simulation was performed using a parallel version of the CTH shock-physics code on the 1840-processor Intel Paragon at Sandia National Laboratories. 


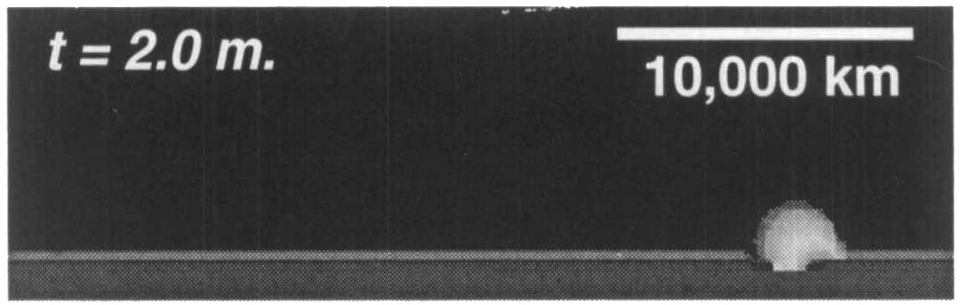

$$
t=5.3 \mathrm{~m} \text {. }
$$
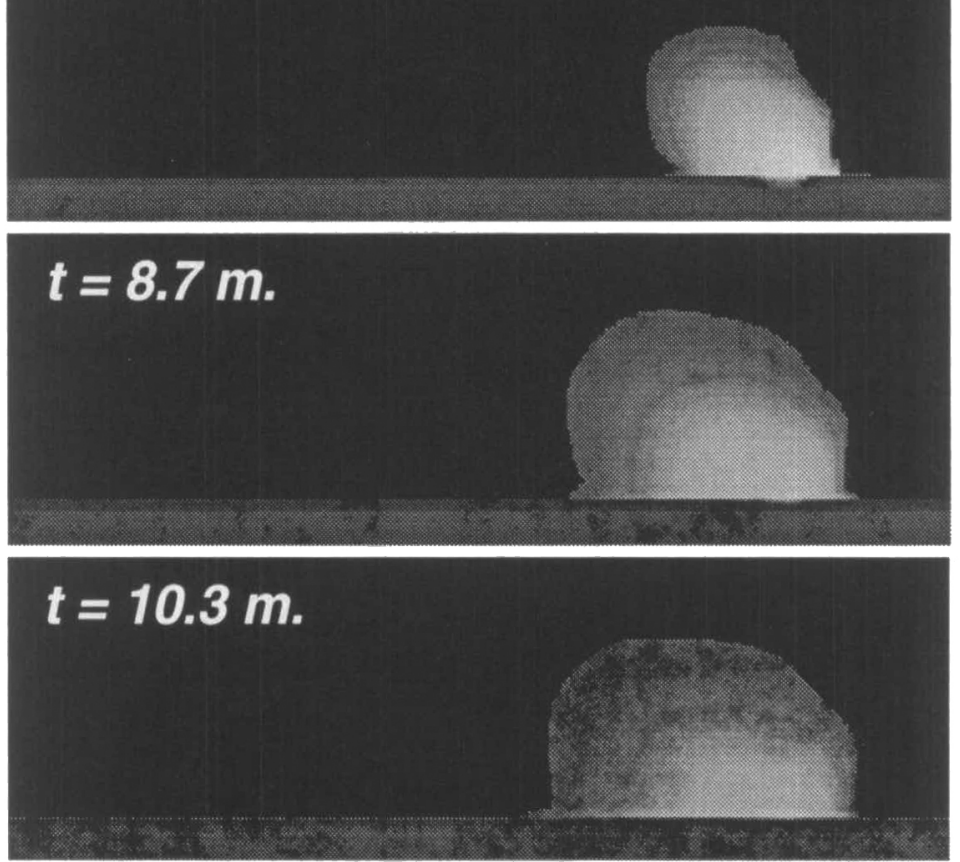

$$
t=18.7 m . \quad \text { 2 } \log (\text { density })
$$

FIgURE 2. Post-impact computational simulation of 3-D fireball and plume evolution after the impact of a $3-\mathrm{km}$ diameter fragment. Shading indicates $\log$ (density) with a visibility cutoff at $10^{-12} \mathrm{~g} \mathrm{~cm}^{3}$. Here, the initial conditions and driving physics are the same as the pre-impact simulation but the spatial resolution has been reduced to allow the simulation to span a longer event time. The inelastic collision of the plume splashback (most dramatically illustrated at the end of the simulation) yields enough thermal heating over a broad area to account for the strength of the 'main' infrared event (from Boslough et al. 1995). 


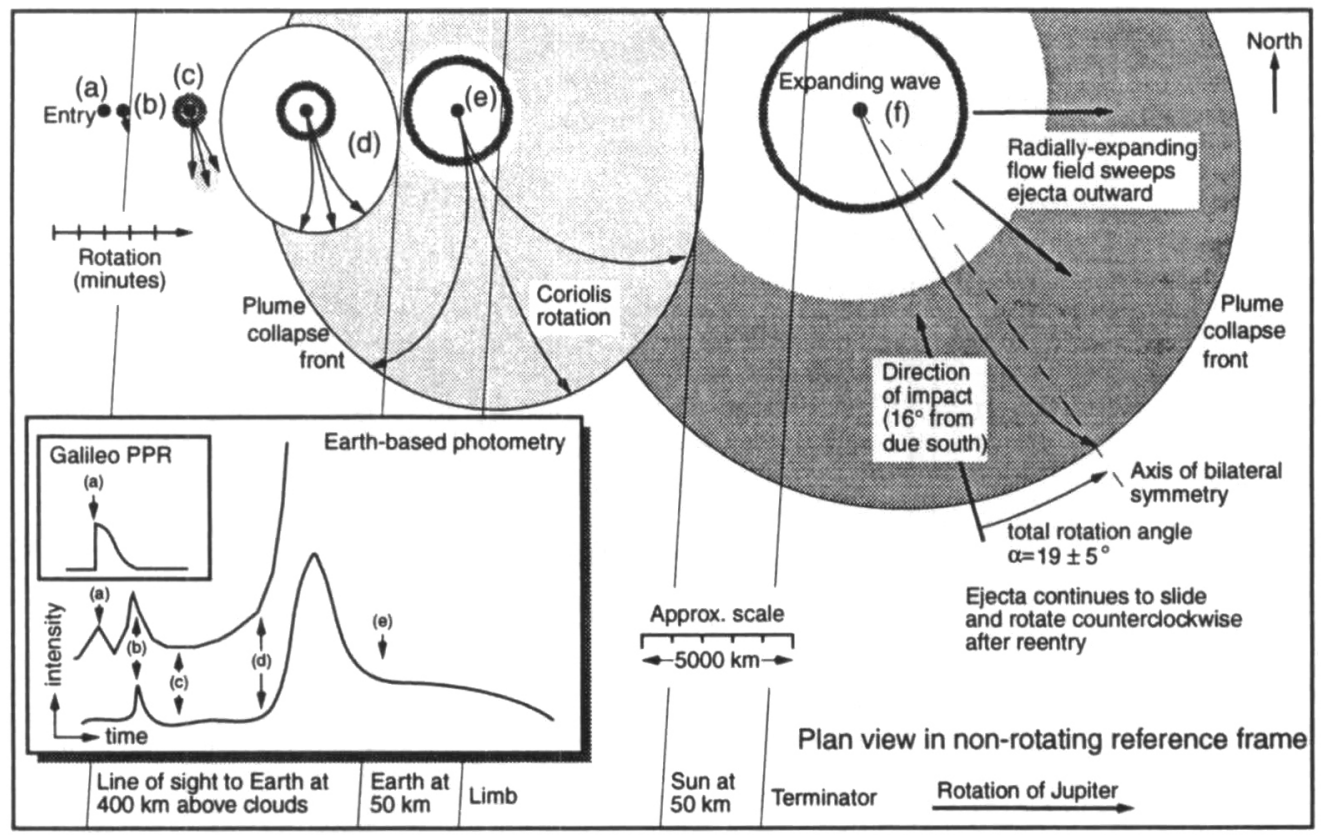

FIGURE 3. Plan view (map projection) of idealized impact site from a stationary (non-rotating vantage point, with snapshots of a planar projection of its evolution. This figure schematically represents features that were seen after several of the larger impacts and depicts an interpretation based, in part, on computational simulations (from Boslough et al. 1995).

is a composite of features observed from various events. It depicts an overhead view of an impact site from a non-rotating vantage point with map projections of the evolving impact sites at various times after impact.

Of course within the context of a qualitative theoretical framework, detailed questions still remain. For instance, how deeply did the fragments penetrate into the atmosphere? There is observational evidence that the largest fragments penetrated the $\mathrm{NH}_{4} \mathrm{SH}$ layer but did not penetrate as far as the Jovian water table (Zahnle, this volume). This is a relatively narrow range of possible penetration depths but, what mass and/or size fragments will reach these depths? This is where our theoretical understanding is most vague. Aside from our poor understanding of the composition and mechanical properties of the comet fragments (after all, it is our hope that the impact of SL9 on Jupiter will help provide some of this information), the answer to this question depends on the approximations that must be made.

Most thcoretical models of the SL9 impact usually divide the problem into at least two, perhaps more, phases. The first phase describes the first 10-30 seconds of the impact as a fragment penetrates the atmosphere. The second describes the early development of the fireball up to several minutes after impact. Sometimes, a third phase of the problem, the plume splashback, is analyzed. Each part of the problem presents unique challenges that require approximation in some form or another. Some modelers combine different parts of the problem or treat the same part in different ways to perform 'reality checks'. 
The approximations used in modeling the penetration phase are discussed later in this chapter and in the chapter by MacLow. The plume splashback phase is discussed in the chapter by Zahnle. Perhaps of special note are the approximations required to model the early development of the fireball. Because the fragments penetrated the atmosphere at an angle of 45 degrees, the problem is intrinsically three dimensional. While a limited number of 3D calculations have been performed on supercomputers (e.g., Figure 1 and the simulations of Takata et al. 1994), it is simply more practical to explore model dependences using two-dimensional approximations. There are two possible endmember $2 \mathrm{D}$ approximations that can be used. One is to approximate the fireball as resulting from a vertical impact and exploit cylindrical symmetry about the vertical axis (e.g., Zahnle \& MacLow, 1994). Another, which we will exploit later in this chapter, is to use cylindrical symmetry with respect to the entry trajectory (inclined at 45 degrees). Neither approach is completely correct. The real answer lies somewhere in between.

In the remainder of this chapter, an analytical model of fragment penetration into the Jovian atmosphere is derived and calibrated with the knowledge gained from earlier work (Crawford et al. 1995b). A series of detailed two-dimensional fireball calculations are then performed using, as input, the energy deposition curves derived from the analytical model. Plumes resulting from fragments with diameters of $125,250,375,500,750,1000$, 1500 and 2000 meters are presented. The plumes are ballistically extrapolated, their morphology at maximum altitude is presented, and the resulting ejecta patterns are shown.

\section{Entry models}

Understanding the mechanisms of energy loss during meteoroid traversal of planetary atmospheres is crucial for understanding the development of fireballs and plumes that were observed during the impact of Comet Shoemaker-Levy 9. Early-time evolution of the fireball is most dependent on the nature of energy deposition at relatively high altitudes (Boslough et al. 1995; Crawford et al. 1995a), yet final penetration depth is most dependent on energy deposition at relatively low altitudes (Crawford et al. 1994, 1995; MacLow \& Zahnle, 1994; Takata et al. 1994; Zahnle \& MacLow, 1994). Therefore, consideration of energy deposition at all altitudes is an important component for understanding fireball and plume development.

Analytical models of the deceleration, mass loss, hydrodynamic deformation and mechanical breakup of meteoroids during passage through planetary atmospheres have been proposed and refined by many researchers (Ivanov \& Yu, 1988; Zahnle, 1992; Hills \& Goda, 1993; Ceplecha et al. 1993; Sekanina, 1994). Ceplecha et al. (1993) describe the longest standing model, that of classical ablation. Ivanov \& Yu (1988) describe some of the hydrodynamic deformations experienced by meteoroids in atmospheric flight and Zahnle (1992), Hills \& Goda (1993) and Sekanina (1994) attempt to link the classical ablation model with hydrodynamic deformation and fragmentation models.

Here, we describe classical ablation from the point of view of large impactors such as Shoemaker-Levy 9. A modified ablation model, as suggested by Chevalier \& Sarazin (1994) and Field \& Ferrara (1995), satisfies conservation of energy during the ablative process and adds a further refinement to accomodate observations of terrestrial meteors. A new analytic hydrodynamic deformation model, based on an observation of O'Keefe et al. (1994), compares favorably with numerical simulations performed using the CTH shock-physics code (Crawford et al. 1994, 1995a,b). Coupling the new hydrodynamic model to the modified ablation model yields energy deposition curves resulting from fragment penetration through $1200 \mathrm{~km}$ of Jovian atmosphere. 


\subsection{Classical ablation}

The classical ablation model can be expressed as a series of differential equations representing the deceleration and mass loss of a meteoroid entering a planetary atmosphere (after Zahnle, 1992):

$$
\begin{aligned}
m \frac{d v}{d t} & =-\frac{C_{d}}{2} \pi r^{2} \rho v^{2} \\
Q \frac{d m}{d t} & =-\frac{C_{h}}{2} \pi r^{2} \rho v^{3}
\end{aligned}
$$

where $m, v$ and $r$ are the meteoroid's mass, velocity and radius, respectively, $\rho$ is the atmospheric density and $C_{d}$ and $C_{h}$ are drag and heat transfer coefficients, respectively. Equation (2.1) is simply Newton's law of motion, whereas (2.2) represents mass loss due to radiative or frictional heating by the atmosphere. The ablated mass is assumed to rapidly decelerate to zero velocity relative to the atmosphere. The variable $Q$, usually equated to the heat of fusion or vaporization, can be combined with $C_{d}$ and $C_{h}$ to form an ablation coefficient $\sigma=\frac{1}{2} C_{h} /\left(C_{d} Q\right)$ (Ceplecha et al. 1993). Often, an additional equation is prescribed describing the deformation and/or fragmentation of the meteoroid through change of the radius, $r(t)$ (Zahnle, 1992; Chyba et al. 1993; Hills \& Goda, 1993; Sekanina, 1993; Zahnle \& MacLow 1994).

Strictly speaking, equations (2.1) and (2.2) do not conserve energy when accepted values of $C_{d}=1, C_{h}=0.01-0.6$ and $Q=2.5 \times 10^{10} \mathrm{erg} \mathrm{g}^{-1}$ (Biberman et al. 1980; Zahnle \& MacLow, 1994) or $\sigma=0.01-0.2 \mathrm{~s}^{2} \mathrm{~km}^{-2}$ (Ceplecha et al. 1993; Sekanina, 1993) are used. This is evident when one considers that the power delivered to the system by the atmosphere flowing by the fragment at $60 \mathrm{~km} \mathrm{~s}^{-1}, \frac{1}{2} C_{d} \pi r^{2} \rho v^{3}=10^{20} \rho \pi r^{2} \mathrm{erg} \mathrm{s}^{-1}$, is dwarfed by the power represented by loss of 'ablated mass', $\frac{1}{2} \frac{d m}{d t} v^{2}=\frac{1}{2} C_{h} \pi r^{2} \rho v^{5} / Q=$ $10^{23} \rho \pi r^{2}$ erg $\mathrm{s}^{-1}$.

Because the ablated mass is not truly lost from the system until it has been decelerated to a small fraction of the impact velocity (Bronshten, 1983), the correct expression for $Q$ is more appropriately represented by:

$$
Q=Q_{0}+\frac{1}{2} v^{2}
$$

where $Q_{0}$ is equated with the heat of fusion or vaporization in the usual sense and $\frac{1}{2} v^{2}$ is the energy required to accelerate a unit of ablated mass to the velocity $v$. The second term on the right side of $(2.3)$ will dominate when $v^{2} \gg 2 Q_{0}$. For most materials of interest this will occur when $v>5 \mathrm{~km} \mathrm{~s}^{-1}$.

During the impact of Comet Shoemaker-Levy $9\left(v=60 \mathrm{~km} \mathrm{~s}^{-1}\right)$, the second term on the right side of (2.3) was approximately 700 times greater than the first and (2.3) can be closely approximated by

$$
Q \approx \frac{1}{2} v^{2}
$$

This leads to a modified ablation equation (2.2) of the form,

$$
\frac{d m}{d t}=-C_{h} \pi r^{2} \rho v
$$

to be used in conjunction with equation (2.1) and an equation for $r(t)$ to be described later. Field \& Ferrara (1995), using a more sophisticated approach, derive a similar expression and note that $C_{h}$ has a theoretical upper bound of 1.0 .

Equation (2.5) predicts that a 100-m solid ice fragment entering Jupiter's atmosphere will lose less than $1 \%$ of its mass before reaching the 1-bar level. Because this redefinition of $\mathrm{Q}$ implies about 700 times less mass loss, it must be reconciled with the observational 


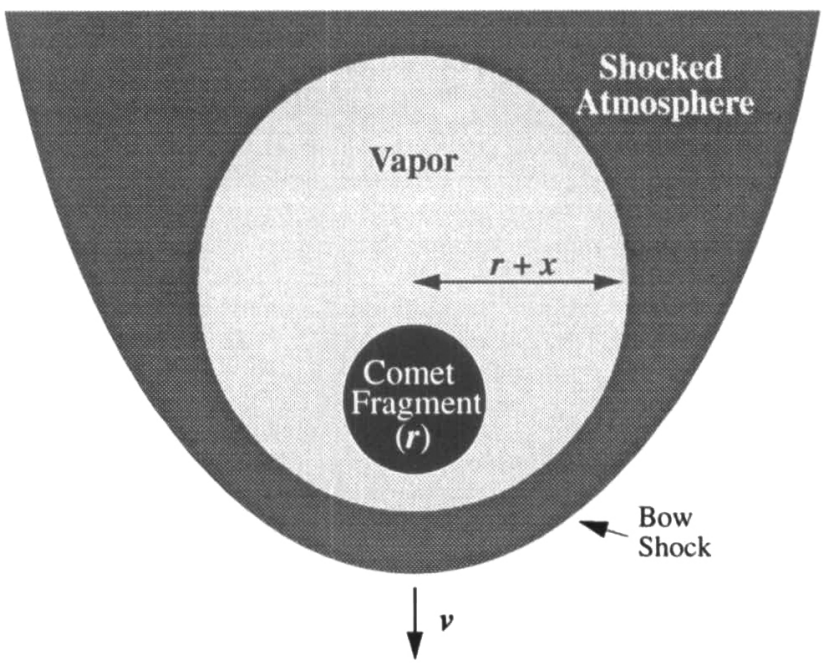

FIgURE 4. Schematic diagram of the analytical entry model that explicitly models the development and behavior of an ablative vapor layer. The increased interaction cross section $\left(S=\pi(r+x)^{2}\right)$ accomodates energy conservation during the ablative process while retaining the large energy deposition rates of previous models. The thickness of the vapor layer, which changes dynamically as the impactor penetrates, is a function of impactor composition, the temperature at the bow shock and the opacity of the vapor and atmospheric gases.

data of small meteors entering Earth's atmosphere-for which (2.2) has been successfully applied many times-before it can be used to describe objects larger than $100 \mathrm{~m}$ entering Jupiter's atmosphere. This, apparently, will be determined by the only remaining free parameter, $r(t)$.

\subsection{Vaporization}

Ceplecha et al. (1993) find two independent parameters for equations 1(2.1) and (2.2): the ablation coeficient $(\sigma)$ and the shape-density coeficient $\left(K=S C_{d} m^{-2 / 3}\right)$ where $S$ is clescribed as the 'head cross-section'. In other words, the 'meteoroid cross-section', $\pi r^{2}$, as portrayed up to now, is actually an 'interaction cross-section' that may include fragments, ablated debris and vapor that is traveling in concert with the main meteoroid during its traverse of the atmosphere. The power deposited in the atmosphere by passage of the meteoroid can be written in terms of $K$ and $\sigma$ :

$$
\frac{d E}{d t}=-\left(m \frac{d v}{d t}+\frac{1}{2} \frac{d m}{d t} v\right) v=K \rho m^{2 / 3}\left(v^{3}+\frac{1}{2} \sigma v^{5}\right) \approx \frac{1}{2} \sigma K \rho m^{2 / 3} v^{5} .
$$

It is clear from (2.6) that a decrease in the accepted values of $\sigma$, as (2.3) and (2.5) imply, can be accomodated by an increase in $K$ to yield the same $d E / d t$. In this case, a hundred-fold increase in $S$ (i.e., a ten-fold increase in $r$ ), for small meteoroids, produces the desired effect.

Consider the dynamics at high altitude, where small terrestrial meteors are observed. At these altitudes, spreading due to aerodynamic forces will be negligible because of the low atmospheric density (Zahnle et al. 1992; Hills \& Goda, 1993). Spreading at these altitudes due to vapor pressure of the ablated meteoroid material can be substantial, however. The ablated vapor traveling in concert with the main fragment occupies a layer of thickness $x$ surrounding the fragment and increases the interaction cross section (Figure 4). 
The mass of the meteoroid vapor layer increases with time as described by equation (2.2) where the original definition of $Q=Q_{0}$, the heat of vaporization, is used. In the meteoroid's reference frame, the bulk of the vaporized mass does not possess the kinetic energy required to escape the immediate vicinity (as shown previously). It will accumulate and form a layer surrounding the meteoroid. Equating the vapor pressure of this layer with the kinematic pressure $\left(\rho^{2}\right)$, provides an expression for the increasing layer thickness $(d x / d t)$ :

$$
\frac{d x}{d t}=\frac{C_{h} R}{8 Q_{0} A} v T_{v}\left(\frac{r}{r+x}\right)^{2}
$$

where $R$ is the molar gas constant, $r$, the meteoroid radius, $A$, the meteoroid mean molecular weight and $T_{v}$, the average vapor temperature. Assuming $T_{v}=10^{4} \mathrm{~K}$ for water ice impacting at $60 \mathrm{~km} \mathrm{~s}^{-1}, d x / d t$ is initially $1 \mathrm{~km} \mathrm{~s}^{-1}$ and the layer will thicken rapidly. At some point, the mass gained by vaporization at the surface of the meteoroid is balanced by mass lost from hydrodynamic stripping at the surface of the vapor layer. Adding a term to account for this produces:

$$
\frac{d x}{d t}=\frac{C_{h} R}{8 Q_{0} A} v T_{v}\left(\frac{r}{r+x}\right)^{2}-\frac{1}{4} \frac{C_{m} R T_{v}}{A v}
$$

where $C_{m}<1$ is the efficiency of mass loss from the vapor layer. The layer will reach an equilibrium thickness, $x_{m}$, when $d x / d t=0$ :

$$
x_{m}=\left(v \sqrt{\frac{C_{h}}{2 C_{m} Q_{0}}}-1\right) r
$$

and $S=\pi\left(r+x_{m}\right)^{2}=\pi r^{2} C_{h} v^{2} /\left(2 C_{m} Q_{0}\right) \approx 150 \pi r^{2}$ for $C_{h}=0.1, C_{m}=0.5, Q_{0}=$ $2.5 \times 10^{10} \mathrm{erg} \mathrm{g}^{-1}$ and $v=60 \mathrm{~km} \mathrm{~s}^{-1}$. For small meteoroids with vapor layers defined by (2.9), it is easy to show that the modified ablation equation (2.5) is equal to (2.2) provided $S=\pi\left(r+x_{m}\right)^{2}$; hence, explicitly modeling the vapor layer in this way reconciles the modified ablation equation (2.5) with the terrestrial observations.

For small meteoroids, $x_{m}$ is important. For large meteoroids $(r>10-100 \mathrm{~m})$, the layer reaches an equilibrium thickness $\left(x_{c}\right)$ when opacity of the vapor is taken into account (Field \& Ferrara, 1995):

$$
x_{c}=\left[\frac{8\left(\gamma_{v}-1\right)(\gamma+1)^{2}}{3 \gamma_{v}(\gamma-1)}\right]^{1 / 2}\left(\frac{\sigma T_{s}^{4} l}{\rho v^{3}}\right)^{1 / 2} r^{1 / 2} .
$$

In this expression, $\gamma_{v}$ and $\gamma$ are for vapor and atmosphere, respectively, $T_{s} \approx 4 \times 10^{4} \mathrm{~K}$ is the shock temperature and $l$ is the photon mean-free-path, which is dependent on vapor temperature and density, generally. From Chevalier \& Sarazin (1994), $l$ is approximately $3 \times 10^{-8} \rho^{-2} \mathrm{~cm}$ for shocked Jovian atmosphere. Since the opacity of vaporized cometary material is considered to be larger than that of clean Jovian air (Chevalier \& Sarazin, 1994; Zahnle \& MacLow, 1994; Field \& Ferrara, 1995), this prescription for $l$ will give an upper bound for $x_{c}$.

\subsection{Hydrodynamic spreading}

Analytical models describing hydrodynamic deformation and mechanical breakup of meteoroids during passage through dense planetary atmospheres have been proposed by several researchers (Ivanov \& Yu, 1988; Zahnle, 1992; Chyba et al. 1993; Hills \& Goda, 1993; O'Keefe et al. 1994). Ivanov \& Yu (1988) modeled the deformations experienced by a fluid spherical body passing through an atmosphere. Zahnle (1992) and Chyba et al. (1993) proposed the 'pancake model' in which the large differential hydrodynamic 

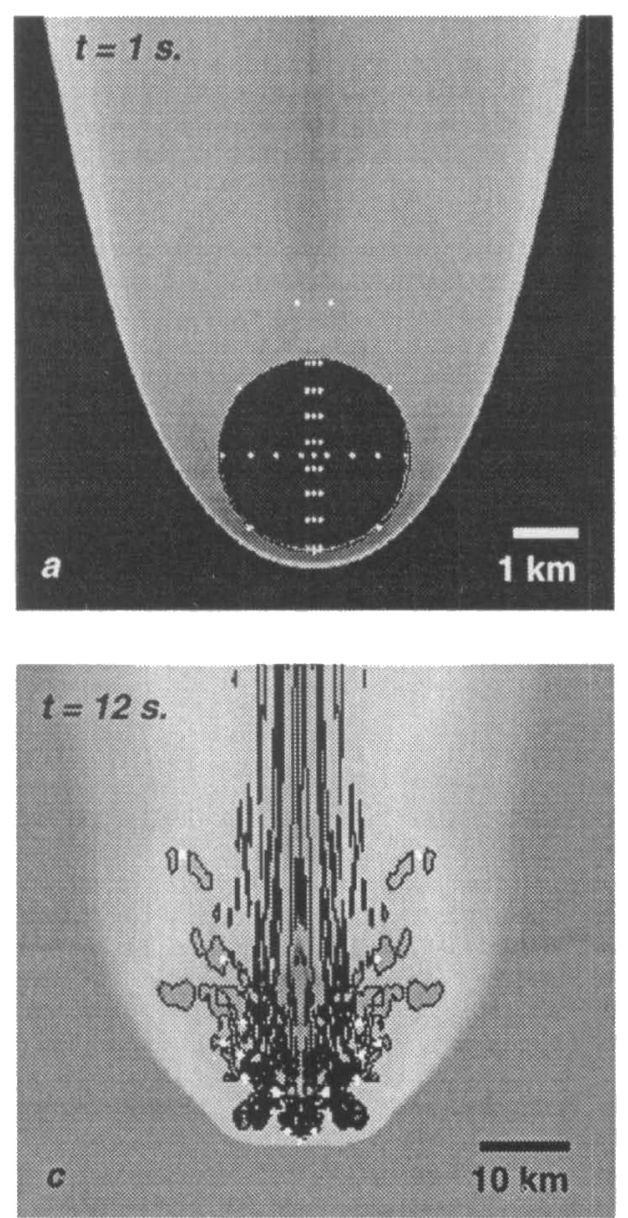

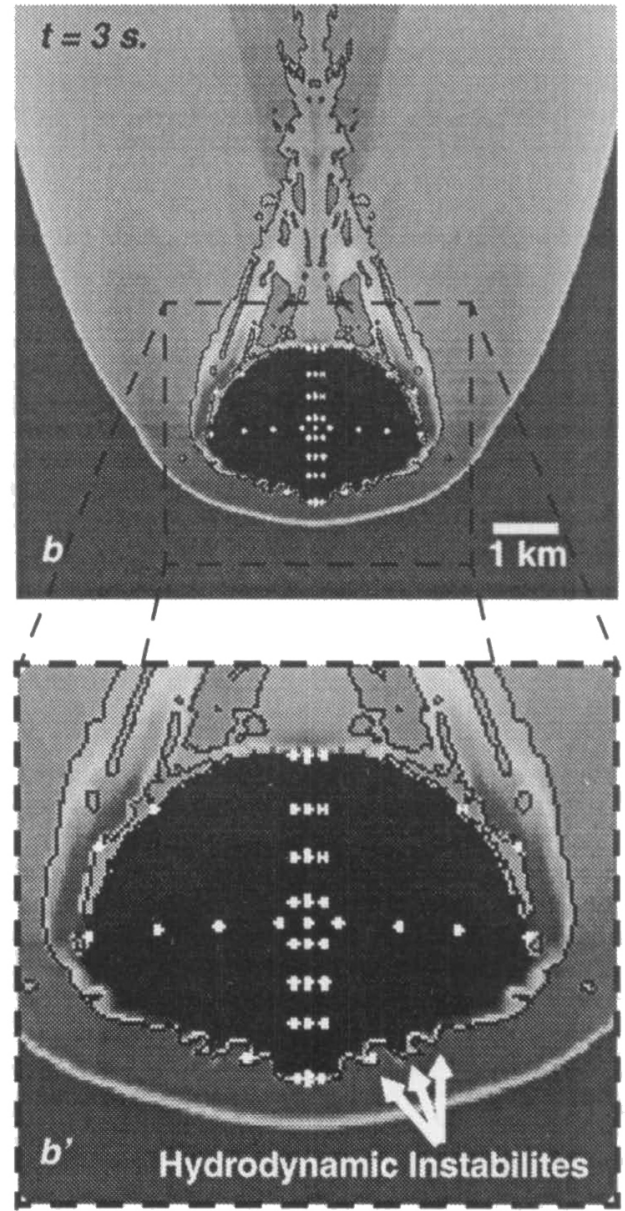

FIGURE 5. Sequential stages of projectile entry (a), deformation (b, $\left.b^{\prime}\right)$ and breakup (c). During entry, the projectile forms a clean bow shock in the upper reaches of the atmosphere. Atmospheric temperatures at the leading edge of the projectile reach values as great as $40,000 \mathrm{~K}$. During deformation, the projectile flattens at an average rate governed by the aerodynamic flow field (the growth rate of a long wavelength Kelvin-Helmholtz instability). Eventually, Rayleigh-Taylor hydrodynamic instabilities fragment the body (after Crawford et al. 1995b).

pressure experienced between the front and rear of an idealized cylindrical projectile is virtually unopposed by the ambient pressure along its sides. The meteroid spreads and flattens, leading to a 'pancake' that rapidly decelerates, depositing the bulk of its energy across less than an atmospheric scale height. Hills \& Goda (1993) proposed a model whereby a small asteroid traveling through an atmosphere suffers successive fragmentation wherever the aerodynamic pressure exceeds the material yield strength. The fragmentation continues until the aerodynamic pressure is lower than the yield strength. Like the pancake model, the spreading meteroid deposits the bulk of its energy across less than an atmospheric scale height. O'Keefe et al. (1994) examined the role of RayleighTaylor (R-T) and Kelvin-Helmholtz (K-H) instabilities on the hydrodynamic deformation and breakup of meteoroids. They proposed that $\mathrm{K}-\mathrm{H}$ instabilities limit spreading and $\mathrm{R}-\mathrm{T}$ instabilities initiate breakup. 
Crawford et al. (1995b) used the shock-physics code CTH to model the entry of 1-km, $2-\mathrm{km}$ and $3-\mathrm{km}$ diameter spherical ice fragments into the Jovian atmosphere (Figure 5). These numerical simulations are best fit by an analytical model based on the idea of O'Keefe et al. (1994). Apparently, the hydrodynamic deformation of fragments of Shoemaker-Levy 9 is controlled by the exponential growth of a long wavelength KelvinHelmholtz instability with spatial wavelength $\lambda=4 r$ where $r$ is the fragment radius. This is the longest wave that a fragment of diameter $2 r$ can support and defines the average behavior of the hydrodynamic spreading. Equating the growth of the amplitude of this wave to the radial growth of the projectile yields:

$$
\frac{d r}{d t}=n r=k_{0}\left(\frac{\rho}{\rho_{f}}\right)^{1 / 2} v
$$

where $n$ is the wave growth rate, $\rho_{f}$ is the bulk density of the fragment and $k_{0}$ is a constant that depends on the dimensionless wavelength, $\lambda / r$ (after Field \& Ferrara, 1995):

$$
k_{0}=\frac{2 \pi}{3}\left(\frac{\gamma-1}{\gamma+1}\right)^{1 / 2} \frac{r}{\lambda} .
$$

For an atmosphere modeled as an ideal gas with $\gamma=1.2-1.4, k_{0}$ has a value of $0.16-0.21$ for the longest supported wavelength.

To compare with the numerical results of Crawford et al. (1995b), who did not model mass loss from radiative ablation, Equation (2.11) is coupled with (2.1) to describe fragment deceleration during entry. A model of the Jovian atmosphere was provided by Orton (unpublished data) and scaled for the 45 degree entry angle. The equations are numerically integrated to find velocity and fragment radius as functions of time and altitude. The deposition of energy by the fragment is found from:

$$
\frac{d E}{d t}=-m \frac{d v}{d t} v
$$

A $k_{0}$ value of 0.2 , consistent with the theoretical arguments above, adequately fits the energy deposition curves derived from the numerical results at least while the long wavelength Kelvin-Helmholtz instability dominates (Figure 6). Eventually, Rayleigh-Taylor instabilities dominate the deformation mode, producing 'outbursts' of spreading and fragmentation. The 'pancake' model (also shown in Figure 6) places the peak explosion altitude slightly higher. Neither model addresses the onset and growth of Rayleigh-Taylor instabilities and, consequently, does not well describe the fragmentation behavior near the terminus of the penetration. Nevertheless, the average energy deposition in the higher altitude region of the Jovian atmosphere appears to be better described by including the limiting effect of the long wavelength Kelvin-Helmholtz instability. It seems that much theoretical, computational and experimental effort will need to be expended in order to advance our understanding of this important process.

\subsection{Putting it all together}

Equations (2.1), (2.5), (2.8) and (2.11) provide a fairly complete theoretical description of the average energy deposition resulting from fragments of Shoemaker-Levy 9 penetrating the higher altitudes (greater than $-100 \mathrm{~km}$ relative to the 1-bar reference level) of Jupiter's atmosphere. Putting the expressions in terms of entry angle $(\theta)$ and altitude in the atmosphere $(z)$ and using the definition for the interaction cross-section, $S=\pi(r+x)^{2}$, yields: 

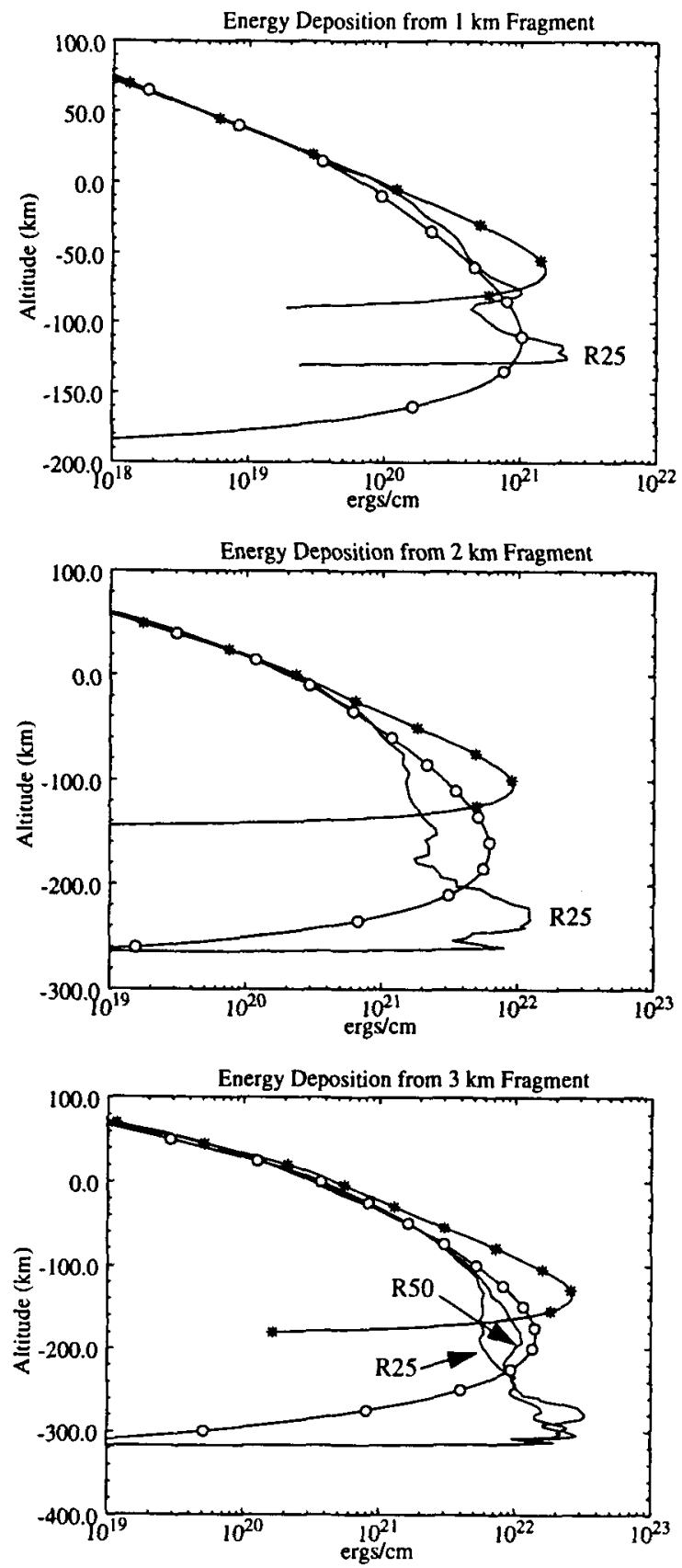

FIGURE 6. Energy deposition curves from 1-, 2- and 3-km fragments entering the Jovian atmosphere. The solid curves without symbols are from numerical simulations of Crawford et al. (1995). The curves are labeled with the resolution of the calculation (represented by the number of zones across the radius, $\mathrm{R} 25$ and $\mathrm{R} 50$ ). The results of our analytical model are superimposed (open circles). The 'pancake' model of Zahnle (1992) places the peak energy deposition slightly higher (stars). 


$$
\begin{gathered}
m_{l} \frac{d v}{d z}=\frac{C_{d}}{2} \pi(r+x)^{2} \rho v \sec \theta \\
\frac{d m}{d z}=C_{m} \pi(r+x)^{2} \rho \sec \theta \\
\frac{d x}{d z=\frac{C_{h} R}{8 Q_{0} A} T_{v}}\left(\frac{r}{r+x}\right)^{2} \sec \theta-\frac{1}{4} \frac{C_{m} R T_{v}}{A v^{2}} \sec \theta \\
\frac{d r}{d z}=k_{0}\left(\frac{\rho}{\rho_{f}}\right)^{1 / 2} \sec \theta
\end{gathered}
$$

where it is understood that for large meteoroids, the thickness of the vapor $(x)$ is limited by (2.10) and mass loss (2.15) is entirely from the vapor layer, as represented by the second term on the right side of (2.16). As the $x_{c}$ limit is approached and the layer becomes optically thick, vapor production at the surface of the meteoroid slows because the surface no longer 'sees' the high temperatures at the bow shock. Below the altitude at which $x_{c}$ is reached, equation (2.16) no longer applies and $x_{c}$ decreases faster than the adiabatic compression of the vapor layer from the kinematic pressure, $\rho v^{2}$. Hence, the compression of the vapor in this altitude region is modeled as:

$$
x=x_{c}\left(\frac{\rho_{c}}{\rho}\right)^{1 / 3 \gamma_{v}}
$$

where $\rho_{c}$ is the atmospheric density at the altitude where $x=x_{c}$. Equations (2.14)(2.18) are numerically integrated and the energy deposition per unit altitude $(d E / d z)$ is:

$$
\frac{d E}{d z}=\left(m \frac{d v}{d z}+\frac{1}{2} \frac{d m}{d z} v\right) v
$$

Figure 7. shows energy deposition curves for representative ice fragments entering the Jovian atmosphere.

\section{Models of fireball/plume evolution}

Why did all the plumes go to the same height? Boslough et al. (1995) suggested that the SL9 fragments were loosely-bound 'rubble piles', possibly with widely varying masses, that dispersed to about the same diameter by the time they reached the atmosphere. This leads to a simple explanation for consistent plume heights but places constraints on the properties of the fragments that cannot be easily accomodated by parent-body breakup models (Asphaug, pers. comm.). Here, we explore an alternative hypothesis. As emphasized in Boslough et al. (1995) and Crawford et al. (1995a), the early-time evolution of the fireball is most dependent on energy deposition at relativly high altitudes in the Jovian atmosphere (greater than $-50 \mathrm{~km}$ relative to the 1-bar reference altitude). Perhaps maximum plume altitude is a direct function of energy deposition in a relatively narrow region of the Jovian atmosphere-from approximately $50 \mathrm{~km}$ below the 1-bar altitude to approximately $50 \mathrm{~km}$ above the 1-bar level, the Jovian tropopause.

The presence of the tropopause is very important to this model. Because the evolution of the fireball is strongly dependent on the density gradient in the atmosphere, and the gradient is greatest at the tropopause, the hot Jovian atmosphere and cometary debris left in this critical region of the atmosphere will accelerate faster than any other. This hypothesis can be tested by performing two-dimensional fireball/plume calculations based on the energy deposition curves derived previously. But first, it is useful to explain why 


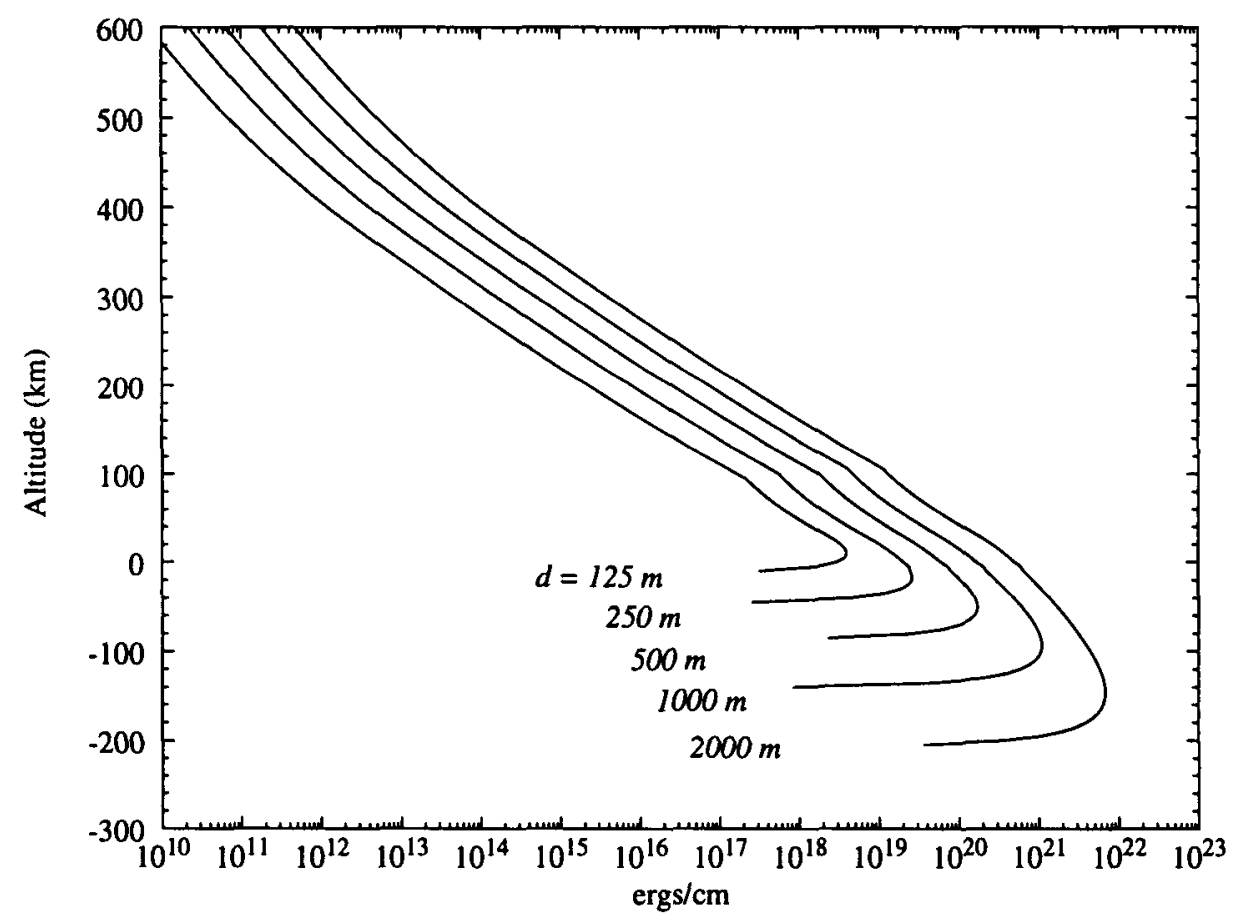

FIgURE 7. Energy deposition curves from our analytical model for $125-2000 \mathrm{~m}$ diameter ice fragments entering the Jovian atmosphere. The change of slope at an altitude of $100 \mathrm{~km}$ results from the opacity limit of Equation (2.10) and appears in about the same location as determined by Zahnle and MacLow (1994).

energy deposited from different size impactors in this 'favored' region of the atmosphere will always yield nearly the same maximum plume altitude.

\subsection{A simple model to explain why all of the observed plumes went to the same height}

Neglecting, for the moment, the interesting question of what happens if a fragment disintegrates and stops in the favored region of the atmosphere, consider the energy deposited by a fragment penetrating through the atmosphere before it has suffered significant deformation. This energy is initially deposited in a column with radius proportional to $r$. The energy deposited per unit length $(d E / d z)$ is

$$
\frac{d E}{d z}=\frac{C_{d}}{2} S \rho v^{2} \sec \theta
$$

where $S$ is the interaction cross-section defined previously. The total energy deposited across a scale height of atmosphere $(H)$ is

$$
E=H \frac{d E}{d z}=H \frac{C_{d}}{2} S \rho v^{2} \sec \theta
$$

into a total atmospheric mass $(M=\rho S H)$. The specific energy $(\epsilon)$ of this column heated by the passage of the fragment is

$$
\epsilon=\frac{E}{M}=\frac{C_{d} v^{2}}{2} \sec \theta
$$


and the characteristic velocity of the heated atmosphere, $u=\sqrt{2 \epsilon}$ (Zel'dovich \& Raizer, $1967)$, is independent of fragment size. Since maximum altitude $\left(Z_{\text {max }}\right)$ will scale as $u^{2} \mathrm{~g}-1$, plumes resulting from different size impactors should, to first order, go to the same height. Hence, the plumes reach the same altitude for much the same reason that a rifle bullet and artillery shell have approximately the same muzzle velocity. The masses of the projectiles are substantially different yet the specific energy of the driving gas is the same.

\subsection{Numerical models of fireball and plume evolution.}

Fireball and plume calculations using the CTH shock-physics computational hydrocode were performed to test the hypothesis of Section 3.1 and to investigate the dynamics of small fragments exploding in the favored region of the atmosphere. CTH is a multimaterial, multi-phase computational shock-physics code that solves mass, momentum and energy conservation and material constitutive relations on an Eulerian grid (McGlaun et al. 1990). It can realistically model equations-of-state of many materials simultaneously. An accurate equation-of-state (including dissociation and ionization) for a mix of $89 \% \mathrm{H}_{2}, 11 \% \mathrm{He}$ and free electrons (Kerley, unpublished data) was used to model the Jovian atmosphere. The atmosphere was constrained to be gravitationally stable with a thermal profile provided by Orton (unpublished data) and extended adiabatically at depth.

Computational simulations of $125,250,375,500,750,1000,1500$ and 2000 meter diameter fragments entering the Jovian atmosphere were performed using a two dimensional cylindrical coordinate system. The resulting fireball retains axisymmetry with respect to the entry channel for much of the early-time plume evolution as described by Crawford et al. (1995b) and as shown in Figure 1. Hence, the atmosphere has been scaled to account for the 45 degree entry angle and the gravitational constant $(\mathrm{g})$ has been reduced accordingly. The resolution of the computational grid varies from $0.6 \mathrm{~km}$ per zone to $2.5 \mathrm{~km}$ per zone for simulations of the smallest and largest impacting fragments, respectively. The energy deposited by each fragment's passage through the atmosphere is based on the analytical model derived in Section 2. This approach has the advantage of simulating the evolving wake and fireball at high altitude even as the fragment is penetrating to lower altitude. The energy of the decelerating and ablating fragment is added to the internal energy of the atmosphere in $2 \mathrm{~km}$ long by $10 \mathrm{r}$ radius cylindrical segments (where $r$ is the fragment radius). Each segment receives the appropriate energy from equation (2.19) at the appropriate time. The simulation starts with the fragment (represented as a source of energy) at $640 \mathrm{~km}$ altitude $(900 \mathrm{~km}$ on the computational grid).

Each simulation has 1000 Lagrangian tracer particles distributed in eight layers of 125 particles each. The topmost layer is located at the approximate location of the Jovian cloud tops (11 km altitude). The remaining layers, in descending order, are at $0,-11$, $-21,-32,-42,-71$ and $-106 \mathrm{~km}$ respectively. The particles are evenly distributed from 0 to $25 \mathrm{~km}$ radially for simulations of large fragments (750 m and larger) and from 0 to $5 \mathrm{~km}$ radially for simulations of small fragments $(500 \mathrm{~m}$ and smaller). These particles trace the ejection of atmospheric materials by the fireball and cooling plume. The locations and velocities of the particles at the end of the simulations are ballistically extrapolated to determine maximum plume height and final ejecta morphology.

Figures 8 and 9 show the first three minutes of entry-wake and plume evolution for 250, 500, 1000 and 2000 meter fragments. In the temperature plots of Figure 8, the penetrating 'fragments' can be followed through the first 20 seconds. Near the end of the penetration phase, the 'entry fireball' is starting to evolve at the top of the entry channel. 


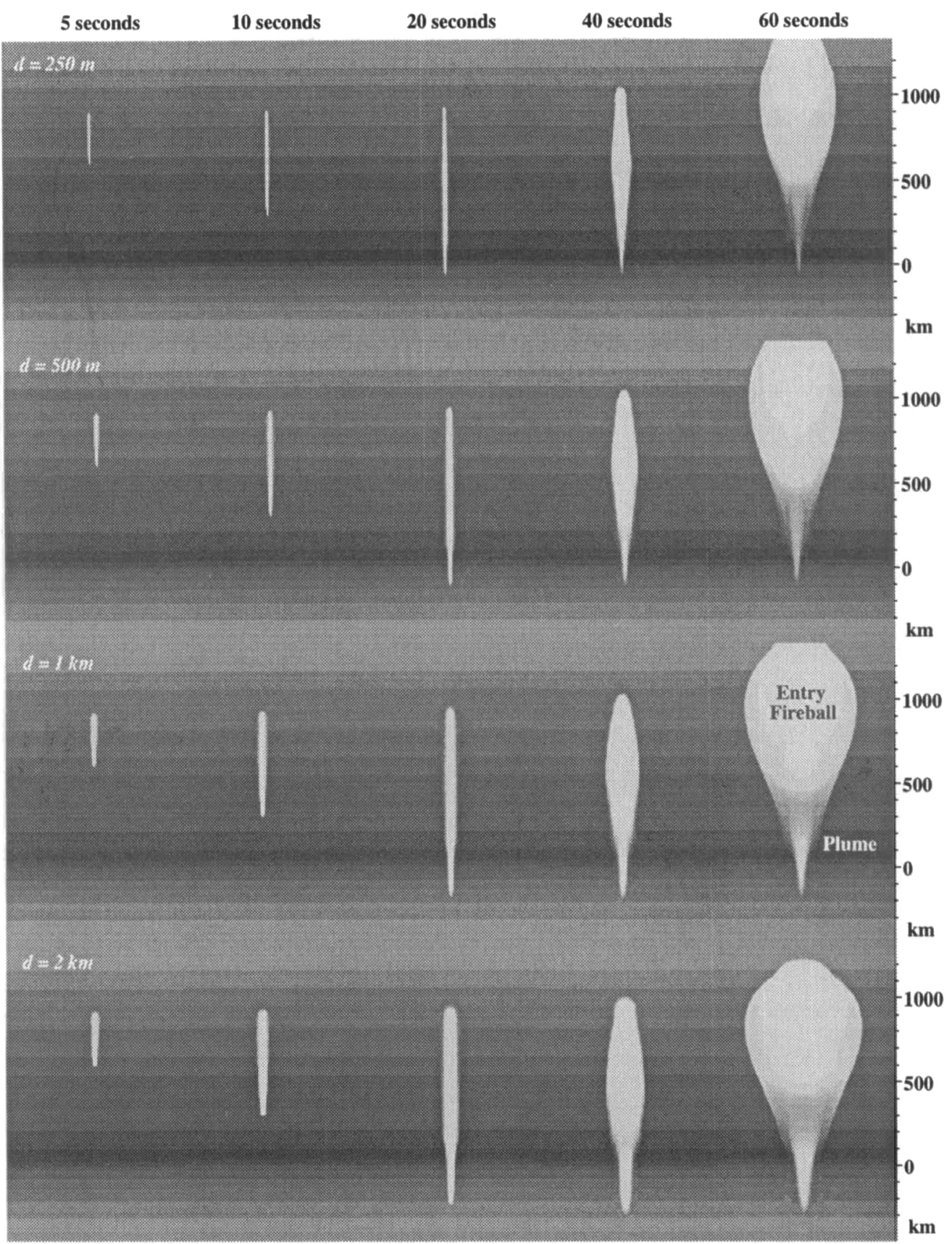

Figure 8. Simulations of the entry of $250,500,1000$ and 2000 meter fragments entering the Jovian atmosphere: the first 60 seconds. Gray scale is proportional to $\log (\mathrm{T})$. The development of the 'entry fireball', beginning at 40 seconds, is clearly seen. The plume can be seen beginning to develop at 60 seconds near the Jovian tropopause. The plume contains opaque materials derived from the cloud layers, hence was probably the over-limb feature observed in the HST images. 


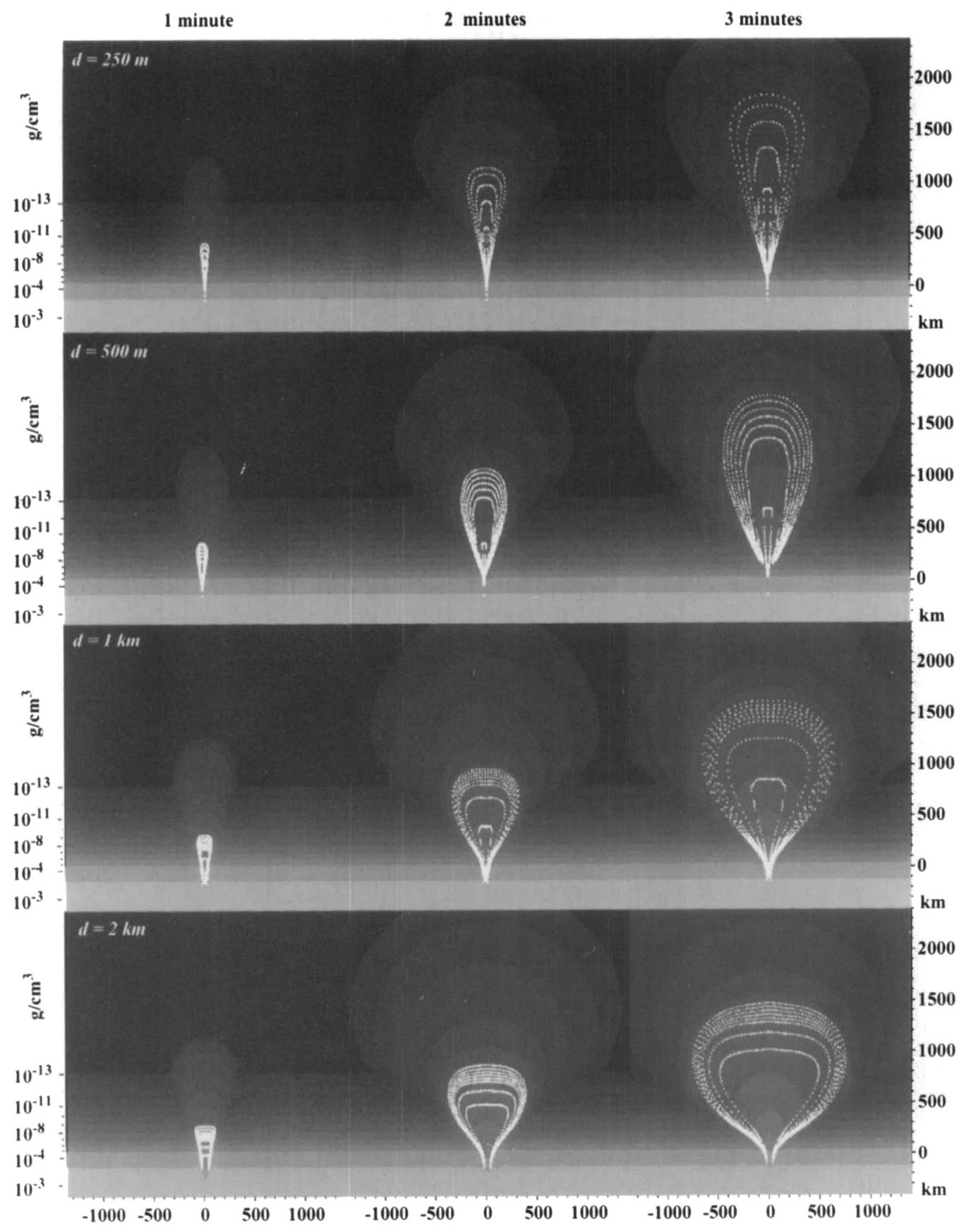

FIGURE 9. Simulations of fireballs from $250,500,1000$ and 2000 meter fragments entering the Jovian atmosphere: the first 3 minutes. Gray scale is proportional to $\log$ (Density). One thousand tracer particles, representing the Jovian cloud layers, are superimposed (white dots). Material derived from the Jovian clouds reach nearly the same altitude at the end of the simulations whereas the isodensity contours do not. 
This fireball has low mass, is probably ionized, and accelerates rapidly out of the Jovian thermosphere at velocities approaching $60 \mathrm{~km} \mathrm{~s}^{-1}$. It could be a perturbing influence on the Jovian magnetosphere, perhaps leading to the increased auroral emissions observed at the magnetic conjugate point of the $\mathrm{K}$ impact site (Clarke et al. 1995). However, the entry fireball is extremely sensitive to the rate of ablation, hence the numbers quoted here should not be taken too literally.

At 40 seconds, the fireball containing the visible plume is seen starting at the tropopause and rising ballistically. No evidence is seen for an 'explosion' at the terminus of penetration although a weak shock can be seen propagating cylindrically. This shock degrades to a linear wave within a few minutes. This is consistent with the model of Chevalier \& Sarazin (1994) and three-dimensional simulations of Crawford et al. (1994) whereby deeply exploding fragments do not generate strong shock blowouts at their terminus (Zahnle \& MacLow, 1994) but generate a buoyant column of hot gas instead.

Figure 9 shows the density distribution of the fireballs and plumes 1,2 and 3 minutes into the simulations. Overlain, are the locations of the eight layers of tracer particles. As is made apparent by the plots, the maximum altitude of a particular isodensity contour strongly increases as a function of fragment size, yet maximum altitude of a given layer of tracer particles (which originate from the same altitude in the atmosphere) does not. As an expression of the lower degree of radial confinement (represented by $H / r$ ), the maximum plume altitude slowly decreases with larger impactor size. Because the plume has cooled to $\approx 100 \mathrm{~K}$ by this time, material derived from the Jovian cloud layers will have condensed. This suggests that the source of opacity for the the plumes was simply the Jovian cloud layers, that the top of the plumes represent a vertical translation of altered cloud materials and that the plume outlined by a layer of tracer particles represents the 'visible plume'. The resolution of the numerical models, and their sensitivity to the rate of ablation do not allow an exact determination of which cloud layer corresponds to the top of the visible plume at this time. However, the morphology of the ejecta (as shown in the next sections) and spectroscopic data (Noll et, al. 1995) suggest that the $\mathrm{NH}_{4} \mathrm{SH}$ layer was probably involved.

The lateral extent and the tracer particle density (i.e., opaque mass density) of the plumes are functions of fragment size. In fact, once the visible plumes are rotated to account for the 45 degree entry angle, the increased lateral extent of the plumes resulting: from larger fragment sizes nearly compensates for the weakly decreasing height of the larger 2-D plumes. For fragments greater than approximately $500 \mathrm{~m}$ in diameter, the mass density of the opaque material (again, represented by the tracers) is proportional to the cross-sectional area of the impactor. This is consistent with a simple model whereby the impactor punches a hole with radius proportional to the fragment radius, $r$, in the Jovian clouds. The contents of the hole make up the visible plume. It is easy to show that for large impactors that penetrate completely through the opaque source layer, the volume of excavated opaque material is proportional $r^{2}$. For small impactors that do not penetrate completely through, but explode inside, the volume of excavated material is proportional to $r^{3}$.

\subsection{Ballistic plume extrapolation and ejecta emplacement}

By the end of the simulations, material located in the upper half of the plume is travelling with little force contribution to its motion except that due to gravity. At this point, the motion of the tracer particles is ballistically extrapolated in three dimensions. Figure 10 shows the configuration of the excavated atmospheric layers at the time of maximum visible plume height. All of the plumes rise to approximately the same maximum altitude, yet the layers within are bunched more tightly together for larger impactors. The former 
is consistent with HST observations (Hammel et al. 1995) and the latter has important implications for the ejecta emplacement process.

Even though the impact angle was 45 degrees from vertical, the line segment connecting the peak of the plume with the impact location is not necessarily 45 degrees from vertical and may actually be an indicator of fragment size. The simulated plume from the largest impacting fragment (2 km diameter) has an inclination angle of just 20 degrees from vertical. This requires a cautionary note, however. Because nature performed this impact experiment in three dimensions and here the simulations were performed in two dimensions, this observation may be qualitatively correct but not quantitatively accurate. For example, the inclination angle of a plume formed by a $2 \mathrm{~km}$ impactor will probably turn out to be somewhat less than 20 degrees. We can guess that this will be the case because the atmospheric density gradient will tend to direct the fireball in a more vertical direction during the early hydrodynamic expansion phase.

As shown in Figure 11, all of the simulated plume heights and times-to-maximumaltitude, except those from the smallest impactor ( $125 \mathrm{~m}$ in diameter), are consistent with measurements (3000 km and 400-600 s, respectively) made by Hammel et al. (1995). If the tops of the plumes originated from the tops of the $\mathrm{NH}_{3}$ cloud layer, then this places a lower bound of $4 \times 10^{12} \mathrm{~g}$ ( $200 \mathrm{~m}$ diameter solid water ice) on the mass of the fragments that caused the A, E, G and W plumes observed by HST. If the plumes originated from the $\mathrm{NH}_{4} \mathrm{SH}$ layer, then masses greater than $4 \times 10^{13} \mathrm{~g}$ (400 $\mathrm{m}$ diameter water ice) are required. Moreover, these mass estimates are consistent with those derived from the SL9 parent body breakup study of Asphaug \& Benz (1994) and average fragment size estimates derived from crater chain measurements on Callisto and Ganymede (McKinnon \& Schenk, 1995).

Figure 12 shows 'ejecta patterns' represented by the extrapolated impact locations of the tracer particles after their re-impact with the Jovian stratosphere at $100 \mathrm{~km}$ altitude. Each plot used the upper six layers of tracer particles (from $-42 \mathrm{~km}$ to $11 \mathrm{~km}$ altitude). The location of each tracer particle was ballistically extrapolated assuming a flat planet approximation with constant $g$ of $2500 \mathrm{~cm} \mathrm{~s}^{2}$. In Figure 12, the relative albedo is proportional to the amount of opaque material produced in the wake of the entering fragment which is assumed to be proportional to the peak temperature $\left(T_{p}\right)$ experienced by the tracer during the numerical simulation. This is represented by the empirical expression:

$$
T_{p}=40,000\left(\frac{r}{r_{t}}\right)^{2} K
$$

where $r_{t}$ is the initial radial location of the tracer particle and $r$ is the radius of the comet fragment. The albedo is an increasing function of the cross-sectional area of the impactor, hence in Figure 12, the relative albedo is scaled by $r^{-2}$ in order to emphasize the morphology of the ejecta.

The simulated ejecta patterns have several features in common with the observed impact sites. They exhibit crescent-shaped ejecta patterns in the uprange direction with a dark central spot and a gap (seen for the larger fragments) in between. The gap appears to be determined by the degree of penetration below the opaque source layer. In the simulated ejecta patterns, the gap appears for fragments greater than $250 \mathrm{~m}$ in diameter, but depends on the choice for the opaque source region (the atmosphere above $-42 \mathrm{~km}$, in this case). A thicker, deeper source region fills in the gap whereas a thinner, shallower one broadens it out.

The simulated ejecta patterns fail to match the observations in one crucial aspect, however. The observed debris patterns were seen to extend for 360 degrees surrounding 

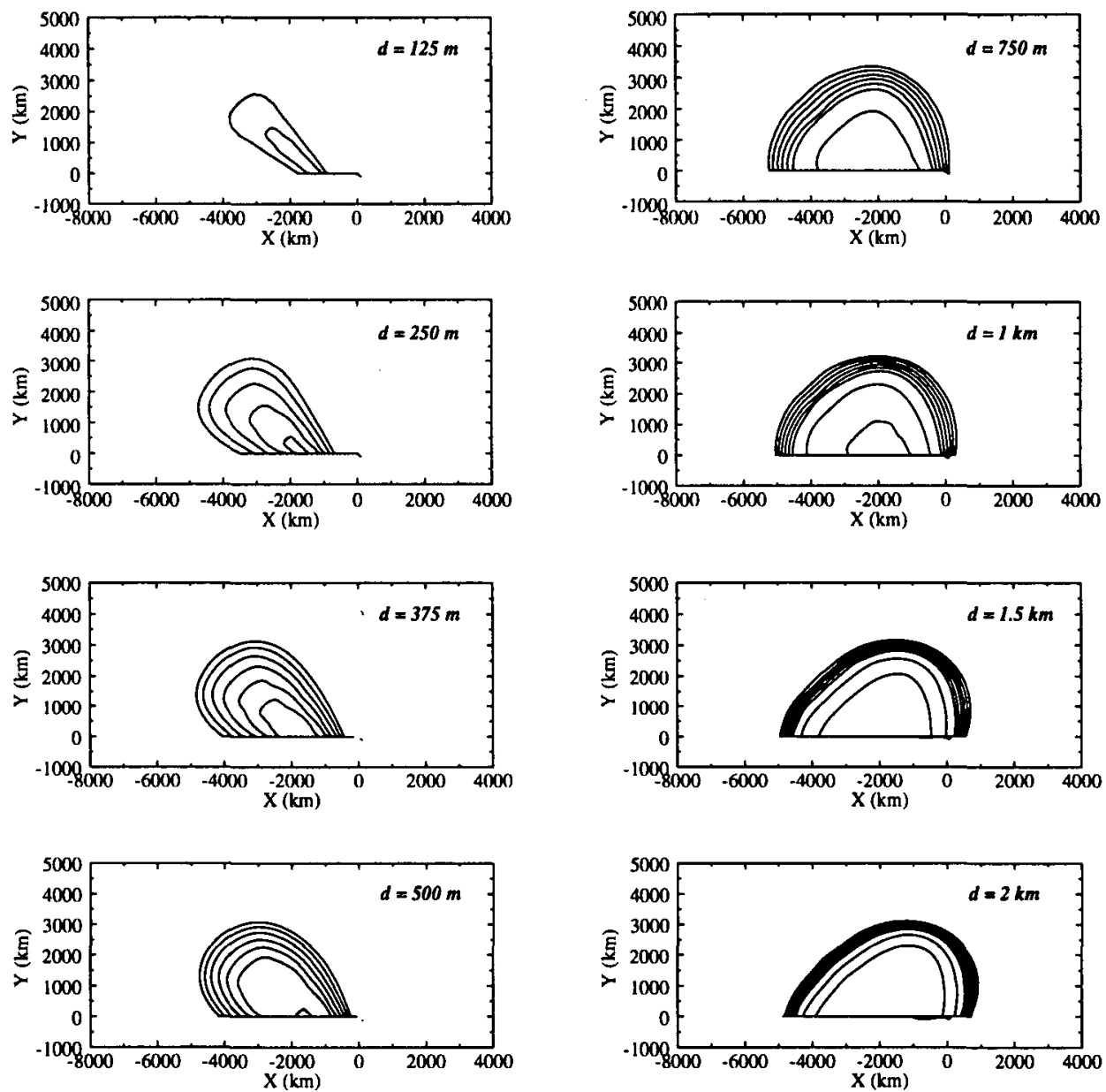

FIGURE 10. Morphology of the simulated plumes arising from the impact of 125-2000 meter diameter fragments entering the Jovian atmosphere. Each plot is made at the time of maximum height with each layer of tracer particles represented by curves in this cross-sectional representation. The topmost layer corresponds to material derived from $11 \mathrm{~km}$ altitude with the remaining layers at $0,-11,-21,-32,-42,-71$ and $-106 \mathrm{~km}$. The origin corresponds to passage of the fragment through the 1-bar level of the atmosphere.

each impact site with a significant crescent-shaped enhancement seen (for the larger impacts) to the south and east. While the crescent-shaped enhancement is produced by the simulations, the surrounding ejecta pattern is not. The disagreement probably arises from the use of a 2D fireball simulation prior to ballistic extrapolation in 3D. As discussed in Section 1, there are only two possible endmember treatments of this problem using 2D simulations. One, is to treat the event as intrinsically vertical, in which case, the ejecta pattern will be constrained to fall in a 360 degree pattern surrounding the impact site but will not produce the crescent-shaped feature that was observed. The other approach, which is used here, is to treat the event as axially symmetric with respect to the impact direction (inclined at 45 degrees in this case), at least for the first few minutes. This approach can simulate the crescent-shaped feature but fails to produce the surrounding ejecta pattern. Obviously, the answer is in between. 

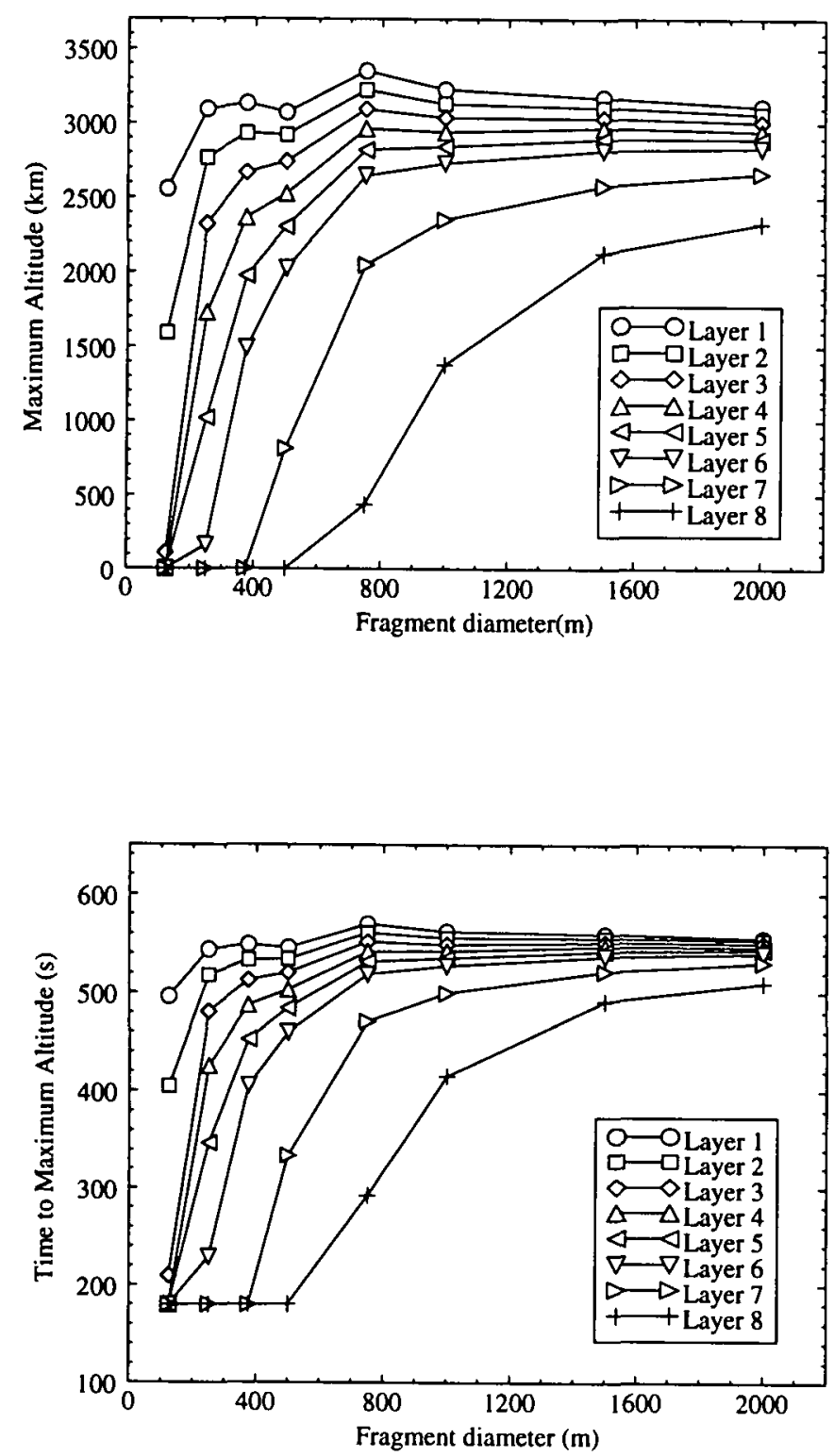

b

Figure 11. Maximum altitude (a) and time-to-maximum-altitude (b) vs. fragment diameter for atmospheric layers represented by tracer particles. Layers 1-8 correspond to initial altitudes of $11,0,-11,-21,-32,-42,-71$ and $-106 \mathrm{~km}$ respectively. 

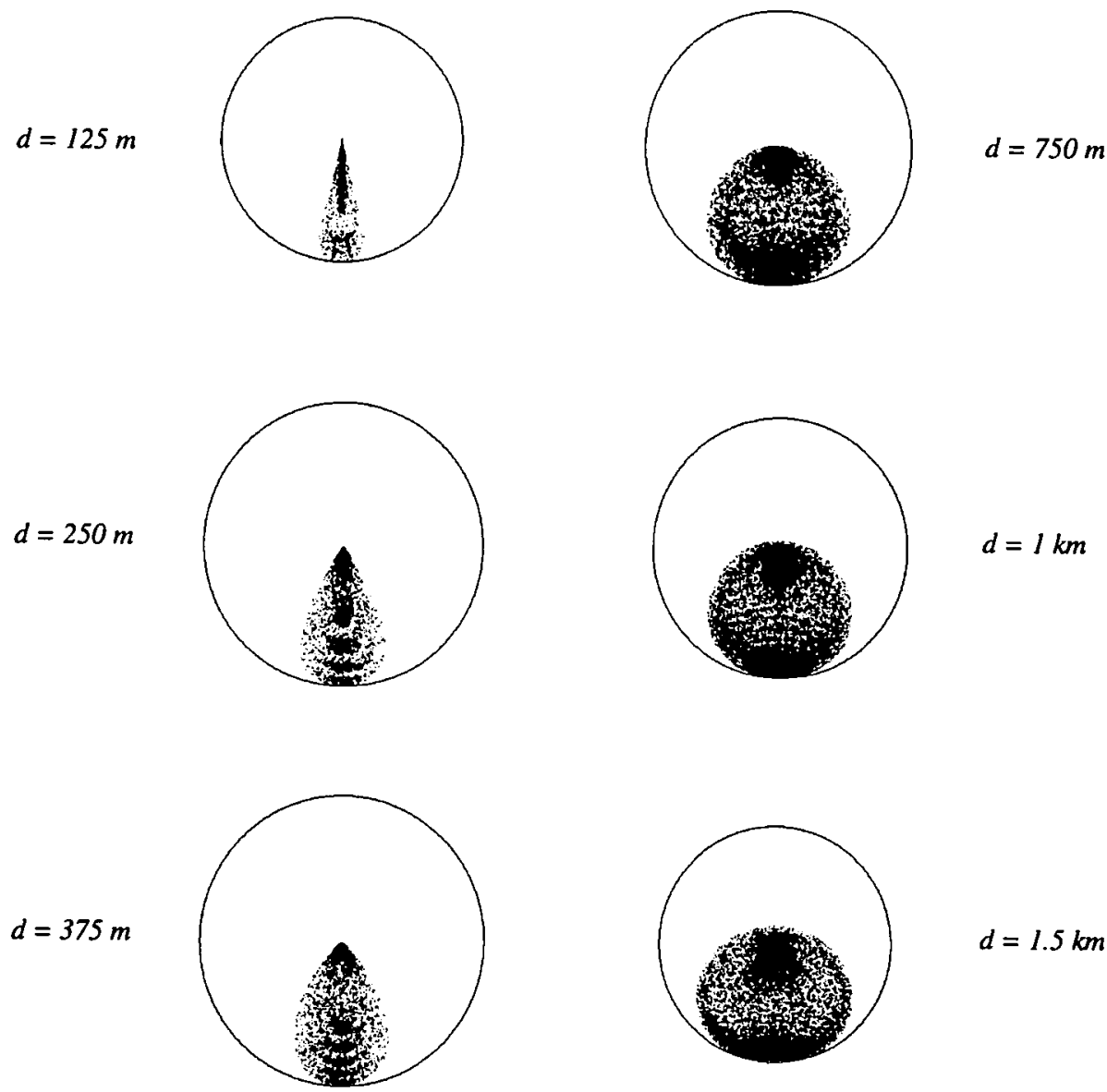

$$
d=1.5 \mathrm{~km}
$$
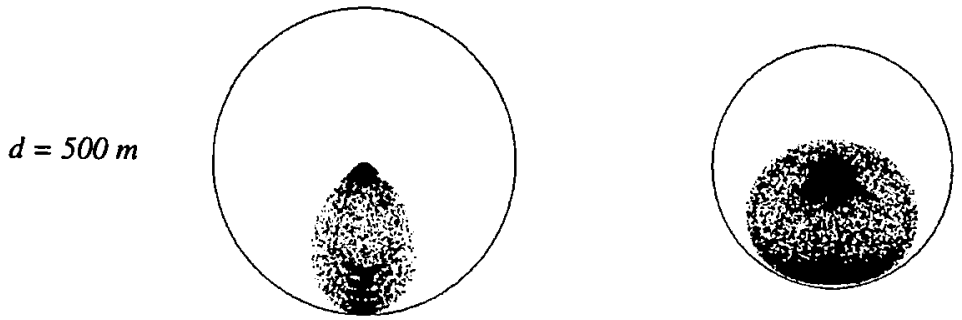

$d=2 \mathrm{~km}$

$10,000 \mathrm{~km}$

FIGURE 12. Ejecta patterns produced by ballistic extrapolation of the uppermost six layers of tracer particles. The albedo of each plot is proportional to peak temperature experienced by the tracers and has been scaled by $d^{-2}$. The circle surrounding each pattern represents the maximum radial extent of ejected material and is centered on the location of fragment passage through the 1-bar level of the atmosphere. 


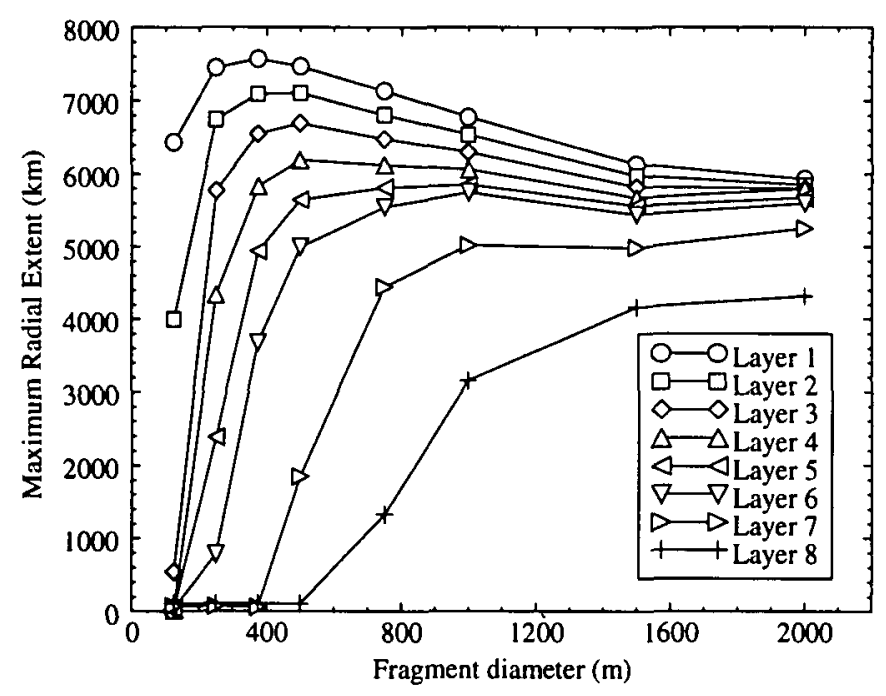

FIGURE 13. Maximum radial extent vs. fragment diameter for atmospheric layers represented by tracer particles. Layers $1-8$ correspond to initial altitudes of $11,0,-11,-21,-32,-42,-71$ and $-106 \mathrm{~km}$ respectively.

The fact that impacts can excavate material normally hidden at depth has been used to understand the history of lunar volcanism (Schultz \& Spudis, 1979) and has been proposed as a model for the impacts of SL9 by Shoemaker et al. (1995). Figure 13 shows the maximum radial extent of material ejected by the plumes of SL9. The eight layers of tracer particles represent a stratigraphic sequence that is mapped out on the surface of Jupiter surrounding an impact site. The radial extents are only about half that required to explain the size of the $G$ impact site, hence significant sliding, as suggested by Boslough et al. (1995), Hammel et al. (1995) and Shoemaker et al. (1995), has probably occurred. In this model, the gap between the crescent and central spot represents a layer in the stratigraphy that is relatively free of opaque source material.

Other processes of opaque production, such as re-entry heating of atmospheric/plume materials during plume collapse (e.g., Boslough et. al. 1995 or Zahnle et al. 1995) or models of gap formation from an evaporation wave (Hammel et al. 1995) can also lead to crescent-shaped features that may be less dependent on the nature of the source region. In any case, opaque material produced in the wake of the penetrating fragments is likely to be a key component of the dark ejecta patterns. This simple process by which wake-synthesized opaque material is subsequently ejected to produce morphologically consistent ejecta patterns is compelling.

\section{Conclusions}

Previous models of radiative ablation and mass loss of bolides traversing Earth's atmosphere can be reconciled with conservation of energy provided that the dynamics of the ablative vapor layer are included. While mass loss due to radiative heating of fragments larger than $100 \mathrm{~m}$ in the Jovian atmosphere is insignificant, ablation is a major contributor to energy deposition at high altitude and has an important role in earlytime fireball evolution. Results from fireball and plume evolution models using the CTH shock-physics code demonstrate that constant maximum plume heights consistent with Hubble observations are observed as long as the tops of the plumes are derived from the 
same level of the atmosphere and that the fragments penetrated at least $30 \mathrm{~km}$ below this level. If the tops of the plumes originated from the visible cloud tops, then fragment masses greater than $4 \times 10^{12} \mathrm{~g}$, corresponding to $200 \mathrm{~m}$ diameter fully dense water ice, are required to explain the observations. If the plumes originated from the $\mathrm{NH}_{4} \mathrm{SH}$ layer, then masses greater than $4 \times 10^{13} \mathrm{~g}$ ( $400 \mathrm{~m}$ water ice) are required. The lateral extent of the plume at maximum height and the darkness and lateral extent of the ejecta pattern are determined by the cross-sectional area of the penetrating fragment. A simple model, whereby opaque material is synthesized in the wake of penetrating fragments and subsequently ejected by the fireball, produces ejecta patterns that are, in many ways, similar to the observed impact sites. A straightforward extension of this model suggests that the apparent gap between the central disturbance of the impact site and the inner front of the crescent-shaped ejecta may reflect the fragment's depth of penetration below the source layer of the visible ejecta.

This chapter benefitted greatly from the leadership and insight of Mark Boslough and from the critical analysis of the other members of Sandia's SL9 team, Tim Trucano and Allen Robinson, and from discussions with Kelly Beatty, Zdenek Ceplecha, Heidi Hammel, Mordecai MacLow, Glen Orton, Toshika Takata and Kevin Zahnle. Gerald Kerley provided the equation of state for Jupiter's atmosphere and Glen Orton provided the structure of the atmosphere based on Voyager data. This work was performed at Sandia National Laboratories under U.S. Department of Energy contract DE-AC04-94AL85000 and was funded by the Laboratory Directed Research and Development Program (LDRD) and the National Science Foundation under Agreement No. 9322118.

\section{REFERENCES}

Asphaug, E. \& Benz, W. 1994 Density of comet Shoemaker-Levy 9 deduced by modelling breakup of the parent 'rubble pile'. Nature 370, 120-124.

Biberman, L. M., Bronin, S. YA. \& Brykin, M. V. 1980 Moving of a blunt body through the dense atmosphere under conditions of severe aerodynamic heating and ablation. Acta Astronautica 7, 53-65.

Boslough M. B., Crawford, D. A., Robinson, A. C. \& Trucano, T. G. 1994a Watching for Fireballs on Jupiter. Eos 75, 305-310.

Boslough M. B., Crawford, D. A., Robinson, A. C. \& Trucano, T. G. 1994b Mass and penetration depth of Shoemaker-Levy 9 fragments from time-resolved photometry. Geophysical Research Letters 21, 1555-1558.

Boslough M. B., Crawford, D. A., Trucano, T. G., \& Robinson, A. C. 1995 Numerical modeling of Shoemaker-Levy 9 impacts as a framework for interpreting observations. Geophysical Research Letters 22, 1821-1824.

Bronshten, V. A. 1983 Physics of Meteoric Phenomena. Reidel.

Ceplecha, Z., Spurny, P., Borovicka, J., \& Keclikova, J. 1993 Atmospheric fragmentation of meteoroids. Astronomy and Astrophysics 279, 615-626.

Chevalier, R. A. \& Sarazin, C. L. 1994 Explosions of Infalling Comets in Jupiter's Atmosphere. Astrophysical Journal 429, 863-875.

Chyba, C. F., Thomas, P. J. \& Zahnle, K. J. 1993 The 1908 Tunguska explosion: atmospheric disruption of a stony asteroid. Nature 361, 40-44.

Clarke, J. T., Prange, R., Ballester, G. E., Trauger, J., Evans, R., Rego, D., Stapelfeldt, K., Ip, W., Gerard, J.-C., Hammel, H., Ballav, M., Jaffel, L. B., Bertaux, J.-L., Crisp, D., Emerich, C., Harris, W., Horanyi, M., Miller, S., Storrs, A., \& WEAver, H. 1995 HST Far-Ultraviolet Imaging of Jupiter During the Impacts of Comet Shoemaker-Levy 9. Science 267, 1302-1307. 
Crawford, D. A., Boslough, M. B., Trucano, T. G. \& Robinson, A. C. 1994 The Impact of Comet Shoemaker-Levy 9 on Jupiter. Shock Waves 47, 47-50.

Crawford, D. A., Boslough, M. B., Robinson, A. C., \& Trucano, T. G. 1995a Dependence of Shoemaker-Levy 9 Impact Fireball Evolution on Fragment Size and Mass. Lunar and Planetary Science 26, 291-292.

Crawford, D. A., Boslough, M. B., Trucano, T. G. \& Robinson, A. C. 1995b The Impact of Periodic Comet Shoemaker-Levy 9 on Jupiter. Int. J. Impact Engin. 17, 253-262.

Field, G. B. \& Ferrara, A. 1995 The Behavior of Fragments of Comet-Shoemaker-Levy 9 in the Atmosphere of Jupiter. Astrophysical Journal 438, 957-967.

Hammel, H. B., Beebe, R. F., Ingersoll, A. P., Orton, G. S., Mills, J. R., Simon, A. A., Choda, P., Clarke, J. T., De Jong, E., Dowling, T. E., Harrington, J., Huber, L. F., Karkoschka, E., Santori, C. M., Toigo, A., Yeomans, D., \& West, R. A. 1995 HST Imaging of Atmospheric Phenomena Created by the Impact of Comet Shoemaker-Levy 9. Science 267, 1288-1296.

Hills, J. G., \& GodA, M. P. 1993 The Fragmentation of Small Asteroids in the Atmosphere. Astronomical Journal 105, 1114-1144.

IVANov, B. A., \& YU, O. 1988 Simple Hydrodynamic Model of Atmospheric Breakup of Hypervelocity Projectiles. Lunar and Planetary Science 19, 535-536.

MacLow, M. M., \& ZahnLE, K. 1994 Explosion Comet-Shoemaker-Levy 9 on Entry into the Jovian Atmosphere. Astrophysical Journal 434, L33-L36.

McGlaun, J. M., Thompson, S. L., \& ElRick, M. G. 1990 CTH - A three-dimensional shock-wave physics code. Int. J. Impact Engin. 10, 351.

MCKinnon, W. B., \& Schenk, P. M. 1995 Estimates of comet fragment masses from impact crater chains on Callisto and Ganymede. Geophysical Research Letters 22, 1829-1832.

Noll, K. S., McGrath, M. A., Trafton, L. M., Atreya, S. K., Caldwell, J. J., Weaver, H. A., Yelle, R. V., Barnet, C., \& Edgington, S. 1995 HST Spectroscopic Observations of Jupiter After Collision of Comet Shoemaker-Levy 9. Science 267, 1307-1313.

O'Keefe, J. D., Takata, T., \& Ahrens, T. J. 1994 Penetration of Large Bolides into Dense Planetary Atmospheres-Role of Hydrodynamic Instabilities. Lunar and Planetary Science 25, 1023-1024.

Schultz, P. H. \& Spudis, P. H. 1979 Evidence for ancient mare volcanism. Lunar and Planetary Science Conf. Proc. 10, 2899-2918.

SEKaninA, Z. 1993 Disintegration phenomena expected during the forthcoming collision of periodic comet Shoemaker-Levy 9 with Jupiter. Science 262, 382.

Shoemaker, E. M., HAssig, P. J., \& Roddy, D. J. 1995 Numerical simulations of the Shoemaker-Levy 9 impact plumes and clouds: A progress report. Geophysical Research Letters 22, 1825-1828.

Takata, T., O'Keefe, J. D., Ahrens, T. J., \& Orton, G. S. 1994 Comet Shoemaker-Levy 9: Impact on Jupiter and Plume Evolution. Icarus 109, 3-19.

ZAHnLE, K. J. 1992 Airburst Origin of Dark Shadows on Venus. Journal Geophysical Research 97, 10,243-10,255.

ZaHnLE, K. \& MacLow, M. M. 1994 The Collision of Jupiter and Comet Shoemaker-Levy 9. Icarus 108, 1-17.

Zahnle, K., MacLow, M. M., Lodders, K., \& Fegley, B., JR. 1995 Sulfur chemistry in the wake of comet Shoemaker-Levy 9. Geophysical Research Letters 22, 1593-1596.

Zel'dovich, Y. B. \& RaIzer, Y. P. 1967 Physics of Shock Waves and High-Temperature Hydrodynamic Phenomena. Academic Press. 